\title{
CHARACTERIZATION OF COMPLETIONS OF UNIQUE FACTORIZATION DOMAINS
}

\author{
RAYMOND C. HEITMANN
}

\begin{abstract}
It is shown that a complete local ring is the completion of a unique factorization domain if and only if it is a field, a discrete valuation ring, or it has depth at least two and no element of its prime ring is a zerodivisor. It is also shown that the Normal Chain Conjecture is false and that there exist local noncatenary UFDs.
\end{abstract}

This article confronts a rather simple question. Given a complete local ring, when is it the completion of an integrally closed domain? The question is trivial for rings of dimension less than two. If we assume dimension at least two, there are several obvious necessary conditions. These are shown in Theorem 1-the complete local ring must have depth at least two and in the mixed characteristic case, the prime integer $p$ cannot be a zerodivisor. Theorem 8 asserts that these conditions are also sufficient. In fact, the integrally closed domain can be chosen to be a unique factorization domain, and Theorem 8 also shows that in the troublesome mixed characteristic case, the prime $p$ will be a prime element in the UFD.

The most promising idea for using this result seems to be the following: Suppose a local ring has property $(P)$ if and only if its completion does. Then we can prove $(P)$ for a large class of rings if we can prove it for UFDs.

The method employed in the construction is quite simple in concept. A quasilocal ring $(R, M \cap R)$ contained in a complete local ring $(T, M)$ is Noetherian and has completion $T$ provided the map $R \rightarrow T / M^{2}$ is surjective and finitely generated ideals of $R$ are closed in the topology induced from $T$. Finding a local ring $R$ with cardinality $\sup \left(\aleph_{0},|T / M|\right)$ and completion $T$ is an easy exercise. First find a subring which maps onto $T / M^{2}$ and then close up all the finitely generated ideals. To make $R$ a domain is somewhat more difficult because it is necessary to avoid nontrivial intersections with associated primes of $T$. If a prime integer $p$ is a zerodivisor or $M$ is a nonzero ideal without regular elements, this is impossible. However, it can be done otherwise and in fact, that result was proved by Lech in [L]. The construction in this article faces an additional complication. It is no longer possible to achieve our goal by making certain intersections trivial. However, we can force prime divisors of principal ideals to be principal and this is enough to get the desired result.

Finally, we note some repercussions these results have for the various chain conjectures. A 3-dimensional local UFD is necessarily catenary. If $T$ has

Received by the editors February 15, 1991.

1980 Mathematics Subject Classification (1985 Revision). Primary 13B35, 13F15, 13 E99. 
minimal primes of different coheights, it will not satisfy the second chain condition. This provides a counterexample to the Normal Chain Conjecture and so implies the falsity of all conjectures in Chapter 3 of [R2]. In Theorem 10, we construct a local UFD of dimension 4 which is not catenary, answering another question of Ratliff [R2, p. 94].

Notation and conventions. All rings will be commutative with unity. We will sometimes say $(T, M)$ is a quasi-local ring; this means that $T$ is a quasi-local ring with maximal ideal $M . \widehat{T}$ will denote the completion of $T$ in the $M$-adic topology.

Recall that a Krull domain is an intersection of discrete valuation rings on its quotient field with the property that every nonzero element is a unit in all but finitely many valuation rings [B, p. 480]. The essential valuations of a Krull domain are its localizations at height 1 primes. If we express a Krull domain as an intersection of discrete valuation rings with the finiteness property, we must include all essential valuation rings in the intersection. It quickly follows that the intersection of two Krull domains with the same quotient field is again a Krull domain; the essential valuations of the new Krull domain must be a subset of the union of the two sets of essential valuations.

We will use two equivalent definitions of unique factorization domains. In addition to describing a UFD as a domain in which every nonzero nonunit is uniquely expressible as a product of irreducible elements, we also note that a UFD is a Krull domain in which every height 1 prime is principal [B, p. 502]. Two simple observations will be used in the proofs. If the Krull domain $R$ becomes a UFD after a set of principal primes is inverted, $R$ was already a UFD. If $R$ is a UFD, so is $R[X]$ where $X$ is an indeterminate.

We will use $|B|$ to denote the cardinality of the set $B$.

We begin with a rather elementary result. It shows that the result obtained in Theorem 8 is optimal.

Theorem 1. Let $R$ be an integrally closed local domain. Then no integer is a zerodivisor in $\widehat{R}$. Moreover, $\widehat{R}$ is either a field, a discrete valuation ring, or a ring with depth at least two.

Proof. Since $\widehat{R}$ is a faithfully flat extension of $R$, regular elements of $R$ cannot become zerodivisors in $\widehat{R}$. Since the image of the integers in $\widehat{R}$ must be contained in $R$, the first conclusion is clear. If $R$ is a field or a discrete valuation ring, then so is $\widehat{R}$. So we may assume $\operatorname{dim} R>1$. Then it must satisfy Serre's condition (S2) [M, p. 125]. Thus depth $R \geq 2$. As depth $\widehat{R}=$ depth $R$, the proof is complete.

Now we begin the process of showing that any complete local ring which satisfies the conclusion of Theorem 1 is the completion of an integrally closed domain; in fact, it is the completion of a unique factorization domain.

Lemma 2. Let $T$ be a complete local ring with maximal ideal $M, C$ be a countable set of primes in $\operatorname{Spec} T$ such that $M \notin C$ and $D$ be a countable set of elements of $T$. If $I$ is an ideal of $T$ which is contained in no single $P$ in $C$, then $I \not \subset \bigcup\{(P+r) \mid P \in C, r \in D\}$.

Proof. We set up a countable enumeration, i.e., a correspondence with the positive integers, of the set $C \times D$. We will define an increasing function $q(n)$ and 
a sequence of elements $\left\{u_{i}\right\} \subset I$ such that $u_{n+1}-u_{n} \in M^{q(n)}$ and if $(P, r)$ is the $n$th pair in $C \times D, u_{n} \notin P+r+M^{q(n)} .\left\{u_{i}\right\}$ will clearly be a Cauchy sequence and its limit will be in $I$ and outside the specified union.

For convenience, set $u_{0}=0$ and $q(0)=0$. Assume $u_{n}$ and $q(n)$ have been defined. Let $(P, r)$ be the $(n+1)$ th pair. If $u_{n} \notin P+r$, pick $u_{n+1}=$ $u_{n}$. Otherwise, since $I M^{q(n)} \not \subset P$, we can find $y_{n} \in I M^{q(n)}-P$. Here, let $u_{n+1}=u_{n}+y_{n}$. Since $u_{n+1} \notin P+r, u_{n+1} \notin P+r+M^{N}$ for some $N$. Let $q(n+1)=\sup \{q(n)+1, N\}$.

Lemma 3. Let $(T, M)$ be a local ring. Let $C \subset \operatorname{Spec}(T)$, let $I$ be an ideal such that $I \not \subset P \quad \forall P \in C$, and let $D$ be a subset of $T$. Suppose $|C \times D|<|T / M|$. Then $I \not \subset \bigcup\{(P+r) \mid P \in C, r \in D\}$.

Proof. We first prove $I \not \subset \bigcup\{P \in C\}$. It suffices to show $I \not \subset \bigcup\{P+I M \mid P \in$ $C\}$. By Nakayama's lemma, $I \not \subset P+I M$ for any $P \in C$. Letting $V=I / I M$, we have reduced the problem to showing that if a finite-dimensional vector space $V$ over $T / M$ is the union of $|C|$ subspaces where $|C|<|T / M|$, then one subspace must be $V$ itself. No doubt this is well known but the proof is short and so we include it here.

We use induction on $\operatorname{dim} V$, the case of dimension 1 being obvious. If $\operatorname{dim} V=n>1$, write $V=W+(T / M) v$ with $\operatorname{dim} W=n-1$. Fix $w \in W$. Then a subspace cannot contain more than one element of the form $w+\alpha v$ unless it contains both $v$ and $w$; in this case it contains all such elements. Since there are $|T / M|$ choices for $\alpha$ and only $|C|$ subspaces, one subspace must contain both $v$ and $w$ if the union is to contain $V$. It follows that the union of those subspaces which contain $v$ must contain $W$. By the induction assumption, one of these must contain $W$ and so $V$ as well.

To prove the lemma, first find $t \in I-\bigcup\{P \in C\}$. Then, for any $(P, r) \in$ $C \times D, t x \in P+r \Leftrightarrow x \equiv t^{-1} r$ modulo $P$. If $r+P \notin(t+P)(T / P)$, then $t x \notin P+r$. If $t^{-1} r \equiv s$ modulo $P$, we can obtain $t x \notin P+r$ by choosing $x \notin P+s$. Pick one such $s$, if necessary, for each pair in $C \times D$. Let $D^{*}$ be the set of elements chosen. Then $\left|D^{*}\right| \leq|C \times D|<|T / M|$. This means we can choose $x \in T$ such that $x \not \equiv s$ modulo $M$ for any $s \in D^{*}$. Clearly $x \notin \bigcup\left\{(P+s) \mid P \in C, s \in D^{*}\right\}$ and so $t x \in I-\bigcup\{(P+r) \mid P \in C, r \in D\}$.

Definition. Let $(T, M)$ be a complete local ring and let $(R, M \cap R)$ be a quasi-local unique factorization domain contained in $T$ satisfying:

(i) $|R| \leq \sup \left(\aleph_{0},|T / M|\right)$ with equality only if $T / M$ is countable,

(ii) $Q \cap R=(0)$ for all $Q \in \operatorname{Ass}(T)$, and

(iii) if $t \in T$ is regular and $P \in \operatorname{Ass}(T / t T)$, then $h t(P \cap R) \leq 1$.

$R$ is called an $N$-subring of $T$.

Lemma 4. Let $(T, M)$ be a complete local ring, $R$ an $N$-subring of $T, c \in R$, $I$ a finitely generated ideal of $R$, and $c \in I T$. Then there exists $S, R \subset S \subset T$, such that $S$ is an $N$-subring of $T,|S|=|R|$, prime elements in $R$ are prime in $S$, and $c \in I S$.

Proof. First we shall note that if $I=y R$ is a principal prime, then $c \in I$. For, in this case, by property (iii) of $N$-subrings, $y R=P \cap R$ for any $P \in \operatorname{Ass}(T / y T)$ and so it suffices to show $c \in P$. But this is clear as $y T \subset P$. In general, let $I=\left(y_{1}, \ldots, y_{n}\right) R$. First suppose $I \subset P$ with $P=a R \neq(0)$. Then $I \subset a R$, 
$c \in a T$, and by the above, $c \in a R$. So $\left\{y_{i} / a, c / a\right\} \subset R$. It clearly suffices to prove the result with $c$ replaced by $c / a$ and each $y_{i}$ replaced by $y_{i} / a$. As $a^{-1} I T$ properly contains $I T$, the Noetherian property of $T$ guarantees that we cannot repeat this process indefinitely. So we can assume $I$ is contained in no such prime. If $n=1$, this forces $I=R$ and so $S=R$ works.

Next we consider the case $n=2$, which is really the crux of the entire paper. $c=t_{1} y_{1}+t_{2} y_{2}$ for some $t_{1}, t_{2} \in T$. Clearly then, $c=\left(t_{1}+t y_{2}\right) y_{1}+\left(t_{2}-t y_{1}\right) y_{2}$ for any $t \in T$. For some carefully chosen $t$, we will let $x_{1}=t_{1}+t y_{2}, x_{2}=$ $t_{2}-t y_{1}$, and let $\stackrel{\circ}{R}=R\left[x_{1}, y_{2}^{-1}\right] \cap R\left[x_{2}, y_{1}^{-1}\right]$. We claim $\stackrel{\circ}{R} \subset T$. For, if $u / v \in$ $\stackrel{\circ}{R}-T$ with $u, v \in T$, then $(v: u) \subset P$ for some $P \in \operatorname{Ass}(T / v T)$. $P$ cannot contain both $y_{1}$ and $y_{2}$; say $y_{1} \notin P$. Then $u / v \in \stackrel{\circ}{R} \subset R\left[x_{2}, y_{1}^{-1}\right] \subset T_{P}$, which is impossible since $(v: u) \subset P$. This establishes the claim. Then let $S$ be the localization of $\stackrel{\circ}{R}$ at $M \cap \stackrel{\circ}{R}$. Noting, for example, that $x_{2}=\left(c-x_{1} y_{1}\right) / y_{2}$, we see that $x_{1}, x_{2} \in \stackrel{\circ}{R} \subset S$ and so $c \in I S$. Assume that we can choose $t$ so that $x_{1}$ will be transcendental over $R$. (Note $x_{1}$ is transcendental $\Leftrightarrow x_{2}$ is.) Then, since $R$ is a UFD, $R\left[x_{1}\right]$ will be also. As noted in the notation section, $\stackrel{\circ}{R}$, being the intersection of two Krull domains, must be a Krull domain. To understand it, we need only discern what its essential valuations are. Since $R\left[x_{1}, y_{1}^{-1}, y_{2}^{-1}\right]=R\left[x_{2}, y_{1}^{-1}, y_{2}^{-1}\right]$ is a localization of each of the two domains intersected, that is, $R\left[x_{1}, y_{2}^{-1}\right]$ and $R\left[x_{2}, y_{1}^{-1}\right]$, the essential valuations of $\stackrel{\circ}{R}$ will be those of $R\left[x_{1}, y_{1}^{-1}, y_{2}^{-1}\right]$ in addition to finitely many others, which are extended from $R$, in which either $y_{1}$ or $y_{2}$ is not a unit. (The essential valuations of $\stackrel{\circ}{R}$ coincide with those of $R\left[x_{1}\right]$ except that those valuations for which $y_{2}$ is not a unit are removed and replaced by the valuations of $R\left[x_{2}\right]$ for which $y_{2}$ is not a unit.) It follows that each height 1 prime of $R$ extends uniquely to a height 1 prime of $\stackrel{\circ}{R}$ and this new prime is principally generated by the same element. Hence $\stackrel{\circ}{R}$ will be a UFD (since the UFD $R\left[x_{1}, y_{1}^{-1}, y_{2}^{-1}\right]$ is the localization $\stackrel{\circ}{R}\left[\left(y_{1} y_{2}\right)^{-1}\right]$ of $\stackrel{\circ}{R}$ and $y_{1} y_{2}$ is a product of prime elements of $\stackrel{R}{R})$. Now let $P \in \operatorname{Spec} T$ be such that $\operatorname{ht}(P \cap R)=1$. Either $y_{1}$ or $y_{2}$ is not in $P$; without loss of generality, assume $y_{1} \notin P$. Now $|R / P \cap R| \leq|R|$ and so its algebraic closure in $T / P$ has cardinality at most $|R|$. Each choice of $t$ modulo $P$ gives a different $x_{2}$ modulo $P$ and so for all but $|R|$ choices of $t$ modulo $P$, the image of $x_{2}$ in $T / P$ will be transcendental over $R / P \cap R$. Let $D_{(P)} \subset T$ be a full set of coset representatives for those choices of $t$ which make $x_{2}$ algebraic. Of course, if $y_{1} \in P$ and $y_{2} \notin P, D_{(P)}$ will consist of choices which make $x_{1}$ algebraic. Now assume $x_{2}$ is transcendental over $R / P \cap R$ as an element of $T / P$. Then $P \cap R\left[x_{2}\right]=(P \cap R) R\left[x_{2}\right]=a R\left[x_{2}\right]$ and it follows that height $(P \cap S) \leq 1$. Also, if we use this procedure for $P \in \operatorname{Ass}(T)$ and $P \cap R=(0)$, we satisfy condition (ii) of the definition of $N$ subring and also guarantee the needed condition that $x_{1}$ will be transcendental over $R$. Let $C=\{P \in \operatorname{Spec}(T) \mid P \in \operatorname{Ass}(T / r T)$ with $0 \neq r \in R\} \cup \operatorname{Ass}(T)$ and $D=\bigcup\left\{D_{(P)} \mid P \in C\right\}$. As $|C| \leq|R|,|C \times D| \leq|R|$ and so, using Lemma 2 if $R$ is countable and Lemma 3 otherwise (both with $I=M$ ), we can choose $t$ to handle all of these primes simultaneously. (Note that $M \in C$ cannot occur as $y_{1}, y_{2} \in M$.) Now, if $P \in \operatorname{Ass}(T / z T)$ for $z$ regular in $T$ and $\operatorname{ht}(P \cap S)>1$, 
then $\operatorname{ht}(P \cap \stackrel{\circ}{R})>1$ and this forces $h t(P \cap R)>0$. However, if $0 \neq r \in P \cap R$, then $P \in \operatorname{Ass}(T / r T) \subset C$ and $\operatorname{ht}(P \cap \stackrel{\circ}{R})=1$ by our choice of $t$. Thus condition (iii) holds and $S$ is the desired $N$-subring.

For $n>2$, we will use a multi-step process. We construct an $N$-subring $R^{\prime \prime}$, $R \subset R^{\prime \prime} \subset T$, such that $\exists c^{*} \in R^{\prime \prime}$ and an $(n-1)$ generated ideal $J$ of $R^{\prime \prime}$ with $c^{*} \in J T$ and whenever $R^{\prime \prime} \subset S$ with $c^{*} \in J S$, then $c \in I S$. Then, by induction, the proof is complete.

Let $I=\left(y_{1}, \ldots, y_{n}\right) R . c=\sum t_{i} y_{i}$ for some $t_{i} \in T$. We can replace $t_{n}$ by $\tilde{t}=t_{n}+\sum_{i=1}^{n-1} u_{i} y_{i}$ for suitable $\left\{u_{i}\right\}$ and adjust the remaining $t_{i}$ 's accordingly. Then, if $R^{\prime}=R[\tilde{t}], J=\left(y_{1}, \ldots, y_{n-1}\right), c^{*}=c-\tilde{t} y_{n}$, and $R^{\prime \prime}$ is the localization of $R^{\prime}$ at $M \cap R^{\prime}$, we are done if we can show $R^{\prime \prime}$ is an $N$-subring. First consider the case $J \not \subset Q$ whenever $\operatorname{ht}(Q)=1$. Here the construction of $R^{\prime}$ resembles the $n=2$ case but is somewhat easier because we are merely adjoining a single transcendental element and so avoid the device of intersecting two different Krull domains. We must choose $\left\{u_{i}\right\}$ so that the image of $\tilde{t}$ in $T / P$ is transcendental over $R / P \cap R$ whenever $P \in \operatorname{Ass}(T / r T)$ for $r \in R$ (including $r=0)$. As in the $n=2$ case, this is enough to force $R^{\prime \prime}$ to be an $N$-subring of $T$. To choose $\left\{u_{i}\right\}$, we ignore those primes which contain $y_{1}$ and follow the $n=2$ procedure to select $u_{1}$ so that $t_{n}+u_{1} y_{1}$ will be transcendental over $R / P \cap R$ as an element of $T / P$ for every other $P \in C$. Then, repeat the process, this time ignoring those primes which contain $y_{2}$ to choose $u_{2}$ so that $t_{n}+u_{1} y_{1}+u_{2} y_{2}$ is transcendental over $R / P \cap R$ whenever $P \in C$ and $y_{2} \notin P$. Now, if $y_{2} \in P$, so is $u_{2} y_{2}$ and so the $P$-residue classes of $t_{n}+u_{1} y_{1}+u_{2} y_{2}$ and $t_{n}+u_{1} y_{1}$ are the same. Thus the $P$-residue class of $t_{n}+u_{1} y_{1}+u_{2} y_{2}$ is also transcendental over $R / P \cap R$ if $y_{1} \notin P$. Adding additional terms does not mess up what was previously achieved. So, continuing on if necessary, we find the desired $\tilde{t}$. On the other hand, if $J \subset Q$ for some ht 1 prime $Q$, we cannot handle that prime and this simple procedure fails. Here, we do have $J=d J^{*}$ for some $d \in R$ and $J^{*} \not \subset Q$ for all such $Q$. We handle this case by combining the $n=2$ case with $I=\left(y_{n}, d\right)$ with the above process where $J=J^{*}$. Start from the equation $c=t_{1} y_{1}+t_{2} y_{2}+\cdots+t_{n} y_{n}=t_{1} d z_{1}+\cdots+t_{n-1} d z_{n-1}+t_{n} y_{n}=$ $t_{n} y_{n}+\left(t_{1} z_{1}+\cdots+t_{n-1} z_{n-1}\right) d=t_{n} y_{n}+w d$, where the elements $w, z_{i}$ are defined transparently by the equation. Then apply the $n=2$ case to obtain an $N$-subring $R^{\prime \prime \prime}$ such that $c=v_{1} y_{n}+v_{2} d$ with $v_{1}, v_{2} \in R^{\prime \prime \prime}$. To obtain $c \in I S$, it suffices to get $v_{2} d \in I S$. For this, it suffices to get $v_{2} \in J^{*} S+y_{n} S$. Since, by the construction, $v_{2}=w-t y_{n}$ with $w \in J^{*} T$ and $t \in T$, and since $J^{*} \not \subset Q$ whenever $\operatorname{ht}(Q)=1$, this is just the previous case with $c=v_{2}$.

Lemma 5. Let $(T, M)$ be a complete local ring with depth $T>1, R$ an $N$ subring of $T$, and $u \in T$. Then there exists $S, R \subset S \subset T$, such that $S$ is an $N$-subring of $T,|S|=\sup \left(\aleph_{0},|R|\right)$, prime elements in $R$ are prime in $S$, and $\exists c \in S$ with $u-c \in M^{2}$.

Proof. Let $R^{\prime}=R[u+t]$ and $S=R_{M \cap R^{\prime}}^{\prime}$, for some $t \in M^{2}$. Just as in the previous proof, we use Lemma 2 or Lemma 3 to choose $t$ so that $S$ is an $N$-subring. The depth condition is necessary to avoid $M \in \operatorname{Ass}(T / r T)$.

Definition. If $R \subset S$ are $N$-subrings of $T$, we say $S$ is an $A$-extension of $R$ if prime elements of $R$ are prime in $S$ and $|S| \leq \sup \left(\aleph_{0},|R|\right)$.

Remark. The extensions given by Lemmas 4 and 5 are $A$-extensions. 
To prove the main theorem, we shall need to apply Lemmas 4 and 5 infinitely often. The next result enables us to do so.

Lemma 6. Let $(T, M)$ be a complete local ring and $R_{0}$ an $N$-subring of $T$. Let $\Omega$ be a well-ordered set with least element 0 and assume either $\Omega$ is countable or $\forall \alpha \in \Omega,|\{\beta \in \Omega \mid \beta<\alpha\}|<|T / M|$. Let $\gamma(\alpha)=\sup \{\beta \in \Omega \mid \beta<\alpha\}$. Suppose $\left\{R_{\alpha} \mid \alpha \in \Omega\right\}$ is an ascending collection of rings such that if $\gamma(\alpha)=\alpha$, then $R_{\alpha}=\bigcup_{\beta<\alpha} R_{\beta}$ while if $\gamma(\alpha)<\alpha, R_{\alpha}$ is an A-extension of $R_{\gamma(\alpha)}$.

Then $S=\bigcup R_{\alpha}$ satisfies all conditions to be an $N$-subring of $T$ except the cardinality condition. $|S| \leq \sup \left(\aleph_{0},\left|R_{0}\right|,|\Omega|\right)$. Further, elements which are prime in some $R_{\alpha}$ remain prime in $S$.

Proof. We replace $\Omega$ by $\Omega^{\prime}=\Omega \cup\{\delta\}$ and $\delta>\alpha$ for all $\alpha \in \Omega$. Let $R_{\delta}=S$. The lemma then follows if we can show that for each $\alpha \in \Omega^{\prime}: R_{\alpha}$ satisfies all conditions to be an $N$-subring of $T$ except perhaps the cardinality condition; $\left|R_{\alpha}\right| \leq \sup \left(\aleph_{0},\left|R_{0}\right|,|\{\beta \in \Omega \mid \beta<\alpha\}|\right)$; and if $\beta<\alpha$, an element which is prime in $R_{\beta}$ remains prime in $R_{\alpha}$. We do this by transfinite induction, the case $\alpha=0$ being trivial.

First note that if it is shown that the three statements in the preceding paragraph hold for $R_{\alpha}$, then the cardinality inequality shows that $R_{\alpha}$ is an $N$ subring unless $\alpha=\delta$. So $A$-extensions are permissible. So assume the inductive hypothesis holds $\forall \beta<\alpha$. If $\gamma(\alpha) \neq \alpha$, it holds immediately for $R_{\alpha}$ by the definition of $A$-extension. So assume $\gamma(\alpha)=\alpha$ and $R_{\alpha}=\bigcup_{\beta<\alpha} R_{\beta}$. Certainly $R_{\alpha}$ is a quasi-local domain. Suppose $x$ is prime in some $R_{\beta}, \beta<\alpha$. If $x$ is not prime in $R_{\alpha}$, then $\exists y, z, w \in R_{\alpha}$ with $y z=x w$ and $y, z \notin x R_{\alpha}$. But then we may choose $\sigma, \beta<\sigma<\alpha$, such that $y, z, w \in R_{\sigma}$ and we see $x$ is not prime in $R_{\sigma}$, a contradiction. So $x$ is prime in $R_{\alpha}$. This gives the third statement. Also, if $w \in R_{\alpha}$ is not a unit, $w \in R_{\beta}$ for some $\beta<\alpha$. Then $w$ can be written as a product of primes in the UFD $R_{\beta}$ and so also as a product of primes in $R_{\alpha}$. Thus $R_{\alpha}$ is a UFD.

Condition (ii) of the definition of $N$-subrings follows trivially from the fact that it is true for each $R_{\beta}, \beta<\alpha$. To prove condition (iii), let $t \in T$ be regular and $P \in \operatorname{Ass}(T / t T)$. If $\operatorname{ht}\left(P \cap R_{\alpha}\right)>1$, clearly $h t\left(P \cap R_{\beta}\right)>1$ for some $\beta<\alpha$. This contradicts the fact that $R_{\beta}$ is an $N$-subring. Lastly we must check the cardinality inequality. Since $R_{\alpha}=\bigcup_{\beta<\alpha} R_{\beta}$,

$$
\begin{aligned}
\left|R_{\alpha}\right| & \leq \sum_{\beta<\alpha}\left|R_{\beta}\right| \leq|\{\beta \in \Omega \mid \beta<\alpha\}| \cdot \sup _{\beta<\alpha}\left|R_{\beta}\right| \\
& \leq|\{\beta \in \Omega \mid \beta<\alpha\}| \sup _{\beta<\alpha}\left(\sup \left(\aleph_{0},\left|R_{0}\right|,|\{\sigma \in \Omega \mid \sigma<\beta\}|\right)\right) \\
& \leq|\{\beta \in \Omega \mid \beta<\alpha\}| \sup \left(\aleph_{0},\left|R_{0}\right|,|\{\beta \in \Omega \mid \beta<\alpha\}|\right) \\
& =\sup \left(\aleph_{0},\left|R_{0}\right|,|\{\beta \in \Omega \mid \beta<\alpha\}|\right) .
\end{aligned}
$$

Lemma 7. Let $(T, M)$ be a complete local ring with depth $T>1$. If $R$ is an $N$-subring of $T$ and $\bar{t} \in T / M^{2}$, then there exists an A-extension $S$ of $R$ such that $\bar{t} \in \operatorname{Image}\left(S \rightarrow T / M^{2}\right)$ and for every finitely generated ideal $I$ of $S$, $I T \cap S=I$.

Proof. First employ Lemma 5 to obtain an $A$-extension $R_{0}$ of $R$ such that $\bar{t} \in \operatorname{Image}\left(R_{0} \rightarrow T / M^{2}\right)$. Let $\Omega=\left\{(I, c) \mid I\right.$ finitely generated ideal of $R_{0}$ and $\left.c \in I T \cap R_{0}\right\}$. Then $|\Omega|=\left|R_{0}\right|$ (the case $I=R_{0}$ shows $\left|R_{0}\right|$ is a lower 
bound for $|\Omega|$ and since the number of finite subsets of $R_{0}$ is $\left|R_{0}\right|$, it is also an upper bound) and so either $\Omega$ is countable or $|\Omega|<|T / M|$. Well-order $\Omega$, letting 0 designate its initial element, in such a way that $\Omega$ does not have a maximal element; then it clearly satisfies the hypothesis of Lemma 6 . Next we recursively define a family $\left\{R_{\alpha} \mid \alpha \in \Omega\right\}$ to satisfy the hypothesis of Lemma 6 . We begin with $R_{0}$. If $\gamma(\alpha) \neq \alpha$ and $\gamma(\alpha)=(I, c)$, then we choose $R_{\alpha}$ to be an $A$-extension of $R_{\gamma(\alpha)}$ given by Lemma 4 such that $c \in I R_{\alpha}$. If $\gamma(\alpha)=\alpha$, choose $R_{\alpha}=\bigcup_{\beta<\alpha} R_{\beta}$. Set $R_{1}=\bigcup R_{\alpha}$. By Lemma 6 and the observation that $\Omega$ is countable or $|\Omega|<|T / M|$, we see that $R_{1}$ is an $A$-extension of $R_{0}$. Also, if $I$ is any finitely generated ideal of $R_{0}$ and $c \in I T \cap R_{0}$, then $(I, c)=\gamma(\alpha)$ for some $\alpha \in \Omega$. So $c \in I R_{\alpha} \subset I R_{1}$. Thus $I T \cap R_{0} \subset I R_{1}$.

We repeat the process to obtain an $A$-extension $R_{2}$ of $R_{1}$ such that $I T \cap$ $R_{1} \subset I R_{2}$ for every finitely generated ideal $I$ of $R_{1}$. Continue recursively to obtain an ascending chain $R_{0} \subset R_{1} \subset \cdots$ such that $I T \cap R_{n} \subset I R_{n+1}$ for every finitely generated ideal $I$ of $R_{n}$. Then, by Lemma $6, S=\bigcup R_{i}$ is an $A$ extension of $R$. Further, if $I$ is a finitely generated ideal of $S$, then some $R_{n}$ contains a generating set for $I$, say $y_{1}, \ldots, y_{k}$. If $c \in I T \cap S$, then $c \in R_{m}$ for some $m \geq n$. So $c \in\left(y_{1}, \ldots, y_{k}\right) T \cap R_{m} \Rightarrow c \in\left(y_{1}, \ldots, y_{k}\right) R_{m+1} \subset I$. Thus $I T \cap S=I$ and the lemma is proved.

Theorem 8. Let $(T, M)$ be a complete local ring such that no integer is a zerodivisor in $T$ and depth $T>1$. Then there exists a local unique factorization domain $A$ such that $\widehat{A} \cong T$ and $|A|=\sup \left(\aleph_{0},|T / M|\right)$. If $p \in M$ where $p$ is a nonzero prime integer, then $p A$ is a prime ideal.

Proof. Let $\Omega=T / M^{2}$, well-ordered so that 0 is its initial element and each element of $\Omega$ has fewer than $|\Omega|$ predecessors. $\Omega$ satisfies the hypothesis of Lemma 6 and we recursively construct a family of rings $\left\{R_{\alpha} \mid \alpha \in \Omega\right\}$ which also satisfies the hypothesis. We let $R_{0}$ be the appropriate localization of the prime subring of $T$-either $\mathbb{Q}, \mathbb{Z}_{p}$, or $\mathbb{Z}_{(p)}$. This is an $N$-subring because $p$ is not a zerodivisor in $T$. Whenever $\gamma(\alpha)<\alpha$, we let $R_{\alpha}$ be an $A$-extension of $R_{\gamma(\alpha)}$ chosen in accordance with Lemma 7 so that $\gamma(\alpha) \in \operatorname{Image}\left(R_{\alpha} \rightarrow T / M^{2}\right)$. If $\gamma(\alpha)=\alpha$ choose $R_{\alpha}=\bigcup_{\beta<\alpha} R_{\beta}$. We claim $A=\bigcup R_{\alpha}$ is the desired example.

We need to show $A$ is Noetherian and $\widehat{A} \cong T$; the remaining conclusions follow immediately from Lemma 6 . From the construction, it is clear (by Lemma 7) that $A \rightarrow T / M^{2}$ is surjective and $I T \cap A=I$ for all finitely generated ideals of $A$. Now let $J$ be any ideal of $A$. Then $J T$ is finitely generated as $T$ is Noetherian and so there exists a finitely generated ideal $I \subset J$ such that $I T=J T$. Then $J \subset I T \cap A=I$ and so $J=I$ is finitely generated. Thus $A$ is Noetherian. Since $(M \cap A)^{n} T=M^{n}$, we get $(M \cap A)^{n}=M^{n} \cap A$. Thus a Cauchy sequence in $A$ which converges to zero in $T$ will also converge to zero in $A$ and $\hat{A} \rightarrow T$ is injective. Finally, $A \rightarrow T / M^{2}$ onto implies $\widehat{A} \rightarrow T$ is surjective.

Now we consider the chain conjectures. Recall that a ring $R$ is catenary if all maximal chains of prime ideals between any two primes $P \subset Q$ of $R$ have the same length. A local domain $R$ satisfies the second chain condition if, for each integral extension domain $S$ of $R, S$ is catenary and all maximal ideals of $S$ have the same height. Many of the conjectures discussed in [R2] have fallen in recent years. The weakest of the conjectures is the Normal Chain 
Conjecture: If the integral closure of a local domain $R$ is catenary, $R$ satisfies the second chain condition. It too is false. (Only a few questions offered in the final chapter of that book remain unsolved.) A shorter exposition of this subject and progress report appears in [R3]. Subsequent to that 1981 paper, several conjectures were disproved by Doering in [D].

Theorem 9. The Normal Chain Conjecture fails.

Proof. Let $T=K \llbracket x, y, z, w \rrbracket /(x y, x z) K \llbracket x, y, z, w \rrbracket$ where $K$ is any field and $x, y, z, w$ are indeterminates. Obtain $A$ via Theorem $8 . T=\widehat{A}$ has two minimal primes, one of coheight 3 and one of coheight 2. Quasi-unmixedness is defined by the equality of these numbers; so $A$ is not quasi-unmixed. Thus $A$ does not satisfy the second chain conjecture [R1, p. 517]. On the other hand, $A$ is a UFD of dimension 3 and so every height 1 prime must have coheight 2. Thus, $A$ is a catenary integrally closed domain which does not satisfy the second chain condition, i.e., it has finite noncatenary integral extensions.

Theorem 10. There exists a noncatenary local UFD.

Proof. Let $T=K \llbracket x, y, z, v, w \rrbracket /(x y, x z) K \llbracket x, y, z, v, w \rrbracket$ where $K$ is a field and $x, y, z, v, w$ are indeterminates. Hereafter, these symbols will denote the corresponding elements of $T$. The ring $A$ given by Theorem 8 will certainly be a 4-dimensional local UFD. For $A$ to be noncatenary, it must have a prime $P$ of height 2 and coheight 1 . It would actually be rather miraculous if a haphazardly-constructed $A$ were catenary but we can guarantee that it is not by altering the construction slightly. $K[v, w]_{(v, w)}$ is an $N$-subring of $T$. We can find $\alpha, \beta \in T$ so that $\alpha v+\beta w+x=c$ can be adjoined safely, then find $\gamma, \delta \in T$ so that we can adjoin $d=\gamma v+\delta w+y$, and lastly find $\theta, \varphi \in T$ and adjoin $e=\theta v+\varphi w+z$. Then we complete the process as before to find $A$. Let $P=(y, z, v, w) \cap A$ and $P^{*}=(x, v, w) \cap A$. Note $c \in P^{*}-P$ and $d, e \in P-P^{*}$, so $P$ and $P^{*}$ are distinct and incomparable. Clearly $P P^{*} \subset(v, w) A$ and as neither is the radical of $(v, w) A$, we must have $P, P^{*}$ are distinct height 2 primes. As $P T=(v, w, y, z) T$, if $P \varsubsetneqq \stackrel{\circ}{P}, \stackrel{\circ}{P} T$ can be contained in no prime except $M$. So $\stackrel{\circ}{P},=M$ and $\operatorname{coheight}(P)=1$.

I would like to thank Colorado State University for the gracious use of their facilities during the preparation of this article.

Note added in proof. The author has extended these techniques to show that $R$ can be chosen to have a one point singular locus. This result will appear in Completions of local rings with a one point singular locus in the Journal of Algebra.

\section{REFERENCES}

[B] N. Bourbaki, Commutative algebra, Hermann, Paris, 1972.

[D] A. M. de Souza Doering, The depth conjecture: a counterexample, J. Algebra 77 (1982), 443-448.

[L] C. Lech, A method for constructing bad Noetherian local rings, Lecture Notes in Math., vol. 1183, Springer-Verlag, Berlin, 1986, pp. 241-247.

[M] H. Matsumura, Commutative algebra, Benjamin, New York, 1970.

[N] M. Nagata, Local rings, Interscience, New York, 1962. 
[R1] L. J. Ratliff, Jr., On quasi-unmixed local domains, the altitude formula, and the chain condition for prime ideals, (I), Amer. J. Math 91 (1969), 508-528.

[R2] Chain conjectures in ring theory, Lecture Notes in Math., vol. 647, Springer-Verlag, Berlin, 1978.

[R3] $\quad$ A brief history of the catenary chain conjectures, Amer. Math Monthly 88 (1981), 169-178.

Department of Mathematics, The University of Texas at Austin, Austin, Texas 78712 E-mail address: heitmann@math.utexas.edu 